\title{
An Apartment-based Metaphor for Intuitive Interaction with Ambient Assisted Living Applications
}

\author{
Sebastian Adam | Kizito Ssamula Mukasa \\ Fraunhofer IESE \\ Fraunhofer-Platz 1 \\ 67663 Kaiserslautern, Germany \\ \{sebastian.adam | kizito.mukasa\} \\ @iese.fraunhofer.de
}

\author{
Kai Breiner | Marcus Trapp \\ Software Engineering Research Group \\ University of Kaiserslautern \\ 67663 Kaiserslautern, Germany \\ \{breiner | marcus.trapp\} \\ @informatik.uni-kl.de
}

\begin{abstract}
Ambient Assisted Living (AAL) aims at supporting elderly people in their daily lives, allowing them to grow old at home. In order to provide easy remote control over the rapidly growing number of assistance services from anywhere in the apartment, many AAL environments offer a universal control device. However, the problem of structuring the numerous services for intuitive usage has not been solved satisfactorily yet. This paper introduces a spatial metaphor for universal control devices to structure available services based on the elderly person's own apartment. We carried out a study with 18 younger elderly people using a prototype to evaluate the appropriateness and acceptance of this metaphor. The results included in this paper show that this apartment metaphor is appropriate and accepted by this main target group of AAL.
\end{abstract}

\section{Categories and Subject Descriptors}

H.5.2 [Information interfaces and presentation]: User Interfaces - Ergonomics, Evaluation, Prototyping; H.1.2 [Models and principles]: User/Machine Systems - Human factors.

\section{General Terms}

Design, Experimentation, Human Factors.

\section{Keywords}

Ambient Intelligence, Ambient Assisted Living, Ubiquitous interactivity, Usability, Metaphor, Navigation structure, Apartment metaphor, Empirical investigation, Accessibility.

\section{INTRODUCTION}

Due to demographic and financial constraints in western nations, it is becoming increasingly difficult to find enough caregivers for the growing number of elderly people. Fortunately, the evolving technology of Ambient Intelligence (AmI) [1], [25], [35] has opened a door to the field of Ambient Assisted Living (AAL) [4], [23], which aims at supporting

(c) The Author 2008.

Published by the British Computer Society elderly people in their daily lives, allowing them to grow old at home, that is, to live in their homes or their familiar community as long as possible instead of being moved to nursing homes.

For this purpose, Ambient Assisted Living includes several categories of support applications, for example, Emergency Treatment, Autonomy Enhancement, and Comfort [16]. While services from the Emergency Treatment category should be able to detect emergencies automatically and thus react proactively, services from the other two categories are reactive and should be used explicitly by the assisted person.

Currently, services in AAL are provided by heterogeneous devices that offer user interfaces (UI) with heterogeneous interaction concepts. Handling this heterogeneity is a serious problem even for young elderly people [26], on whom we are currently focusing our research activities. Young elderly people are characterized as being retired but still active and taking part in social activities. They live in their own homes, where they have to take care of a lot of daily tasks. Even for them, many tasks they used to perform by hand with traditional devices are getting more and more cumbersome. Checking whether all windows and doors are closed before going to bed is only a simple example of this. Therefore, many AAL environments offer a universal control device, which allows controlling all heterogeneous services, such as building automation, remotely from anywhere in the apartment in a unified and homogenous manner. The Personal Universal Controller (PUC) [32] and the Universal Remote Console (URC) [33] are examples of such devices.

However, no appropriate answer has yet been found to the problem of structuring the multitude of services available for intuitive operation and navigation. Already today, there are many services in AAL environments (such as home automation) and the number of supporting services will increase significantly in the near future. To achieve a certain level of usability, the structure of the provided services on the user interface is therefore a crucial issue. Even if much knowledge about "User Interfaces for All" is already available [31], the specificities of AAL mentioned above require deeper investigation of the information and navigation structure in this context.

Therefore, our current research in the AAL domain also deals with the question of how a user interface for a universal control device in AAL should be designed in order to be intuitively usable for elderly people. One promising approach is to design a user interface that matches to the mental model of the user [19]. To reflect the way users act and think in their daily life activities (especially elderly users), we therefore 
developed and evaluated an Apartment Metaphor to make services accessible in an as natural way as possible. The results of this research are described in this paper.

The remainder of this paper is structured as follows: After considering some other metaphors in the next section, we introduce our Apartment Metaphor. Then we describe a prototypical implementation and its evaluation in a comprehensive study with elderly people. The paper closes with an outlook on future work.

\section{SHORT OVERVIEW ON METAPHORS}

An intuitive navigation structure is an important aspect with regard to usability. The navigation structure should allow users to easily find required services (or information / functions) without performing long searches. Thus, a search should be successful after performing only a few clicks. Especially for elderly people, the navigation hierarchy should be as flat as possible to prevent them from getting lost in it [5], [36]. Likewise, broad navigation structures should be avoided. Once the service has been found, it is equally important for the user to remember where to find it the next time she/he needs it. Services should therefore be organized in a way that allows fast navigation and easy remembering. One approach that supports this is the use of appropriate metaphors for structuring information in a way that fits the mental model of the user [22]. Metaphors should bridge the mental gap between the real world and their mapping within an information system. This becomes most important when services are intangible and ambient as in the case of the AAL domain. For this purpose, we investigated existing approaches related to metaphors and navigation structures.

When the first graphical computer display emerged, developing and using metaphors for user interfaces started almost simultaneously. Metaphors help to understand the system in terms of real-world objects the users are already familiar with. Spatial metaphors exploit the extraordinary human ability to organize objects in space, to recall and reason about their locations, and many other space-related cognitive abilities [18].

Probably the most often used type of spatial metaphor is the desktop metaphor. One of the first examples was Xerox Star 8010 [30]. This metaphor is widely used on personal computer operating systems. The major limitation of this virtual desktop is the restricted physical space of the computer screen as compared to a physical desktop. The virtual desktop is significantly smaller and thus restricted in the amount of objects that can be displayed. The most famous concepts used for solving this problem are alternating screen usage, distorted views, large virtual workspaces, and multiple virtual workspaces [18]. The latter is widely used in a variety of window managers for providing a dedicated workspace for performing specific tasks (e.g., emailing, typesetting or programming). Implementations of this concept are, for example, the room metaphor and the book metaphor. The room metaphor is used to semantically provide relationships between the single virtual workspaces [7], [8], [15]. Unlike the book metaphor, the user has an intuitive understanding of the content of the single rooms by the symbolic navigation such as doors.

Tasks to be performed with a system can also be used for structuring by grouping them into abstract task groups (e.g., "copy", "cut", and "paste" in "editing tasks"). Thus, tasks can be hierarchically organized to obtain a navigation structure, which is a popular approach [10]. However, in AAL, the relationship between tasks and the services supporting the tasks has to be taken into consideration. Due to the dynamic appearance and disappearance of services, the resulting navigation structure would not be appropriate for the elderly at the top-level, since a whole task group might disappear.

Studies in such highly dynamical environments have been conducted using the instant-messenger metaphor, to easily implement the appearance and disappearance of task supporting services [20]. However, we consider this concept as not appropriate for our target group of elderly people, because most of them are typically not familiar with dynamic navigation structures that can also change dynamically. .

Basically, the room metaphor meets the structuring and navigation needs of elderly people, since it has been validated that the categorization of elements should be a representation of the real world [28], the information space should be less complex, and elderly people need memory cues as provided by these metaphors [29]. However, our preliminary investigation could not reveal any ongoing or closed work related to the applicability and validation of such metaphors in the domain of AAL. What is mostly done is to simply use the normal desktop metaphor [11].

Moreover, most metaphors are just conceptual: They only provide a framework, but their contents may be different. For example, a room metaphor does not necessarily represent existing rooms, but the subjects are organized in a room-like structure (with doors, windows, etc). Fortunately, the Ambient Intelligence technology provides a chance to map the virtual elements of a metaphor to real physical objects, for example, a room to a concrete room in an apartment.

Therefore, our research described in this paper addresses the question of what such a concrete Apartment Metaphor, as introduced in the next section, could look like and whether it is appropriate for universal control devices in the domain of AAL.

\section{THE APARTMENT METAPHOR}

As AAL applications aim at supporting elderly people in their own apartment by assisting their daily tasks, we consider the elderly person's own apartment as a suitable metaphor. Since they are familiar with their own apartment, such a metaphor should be easy to learn, use, and remember. Moreover, the structure of the apartment remains the same, even when devices and services change. Hence, elderly people will not need to adapt to a new structure. Therefore, our hypothesis is that such a metaphor is appropriate for universal control devices in the AAL domain.

The idea of proposing this metaphor was born from many conversations with elderly people in previous projects. We often recognized that elderly people have a natural association between tasks and devices traditionally used to perform these tasks, and that it is often hard for them to imagine "virtual" devices, e.g., IP phones.

The main concepts of our so-called Apartment Metaphor are therefore tasks, devices and rooms. Tasks are - in our context of AAL - real-life activities an (elderly) person intends to do at home, for instance, making a phone call or closing a window. Following this metaphor, each task is assigned to a device, which people traditionally use for its performance. Of course, nowadays it is, for instance, indeed simple to make a phone call via the Internet using VoIP software. Nevertheless, for most of us, and especially for the elderly, a phone is the 
device associated with the task of calling. This representation of traditional real-world devices is thus an important concept in our metaphor. Continuing this idea, each device typically has a (fixed) place within the owner's home. So, following the Apartment Metaphor, each device is assigned to a room it is traditionally located in. All rooms together finally form the whole apartment. Figure 1 depicts these relationships again: Tasks are performed using a device that is located in a room that is part of the apartment.

The Apartment Metaphor therefore aims at mapping the mental model of an apartment to the structure of a user interface. Just like a real apartment, the resulting virtual apartment consists of several rooms (for example, kitchen, living room, hallway, and bathroom). After "entering" a room all devices allocated to this room can be selected directly. By organizing the user interface in this way, we provide the elderly person with a simple way to perform an intended task: They mentally identify the traditional device and the room it is located in, and then easily navigate the other way around by entering the room and selecting the device virtually (see Figure 2). If they want to make a phone call, for instance, they head for the hallway where their phone is located in, and make the call.

Thus, to perform the intended task, they always need two navigational steps only. As intended for the target group of the elderly, we managed to keep the navigational hierarchy very shallow. Moreover, being a real-world metaphor [34], our approach seems appropriate for the specific needs of this target group.

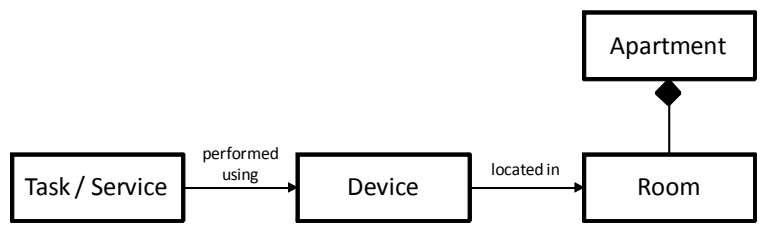

Figure 1. Relationships in the Apartment Metaphor

Finally, in order to deal with the dynamic aspects of AAL systems, we also consider the Apartment Metaphor as a helpful means. As this metaphor is a specific implementation of both room and instant-messenger metaphor, it is also able to reflect the lifetime of an apartment and not only a static snapshot. For instance, when putting a new real device into the room where it belongs, the services provided by this device will be immediately available in the corresponding virtual room on the user interface.

\section{DESIGNING A PROTOTYPE OF THE APARTMENT METAPHOR}

To evaluate the appropriateness of the Apartment Metaphor, we used a prototype technique. Thus, we developed a user interface prototype based on the concepts of this metaphor.

As mentioned before, the main idea of the Apartment Metaphor is not only to represent any apartment, but to represent the user's own apartment (or house), and thus, map his conceptional view of the real environment to the structure of the user interface. When we designed the prototype, this was a challenge, as we were not able to build an individual prototype for each participant of our evaluation.

To cope with this problem, we therefore performed a card sorting [6] study with representatives of our target group to find out a suitable grouping of services in rooms. In this way, we expected to get a wide-accepted structure of services to implement in the prototype in order to avoid any corruption of the future evaluation results. Thus, the purpose of card sorting was just a pragmatic support for our prototype development in order to find an appropriate clustering of items ${ }^{1}$. Indeed, we were sure that there would not be a $100 \%$ overlap between all participants, as, for instance, some people are used to making phone calls in the hallway, while others prefer the living room. However, we estimated a high overlap to enable the evaluation participants to intuitively find most of the devices in the expected rooms.

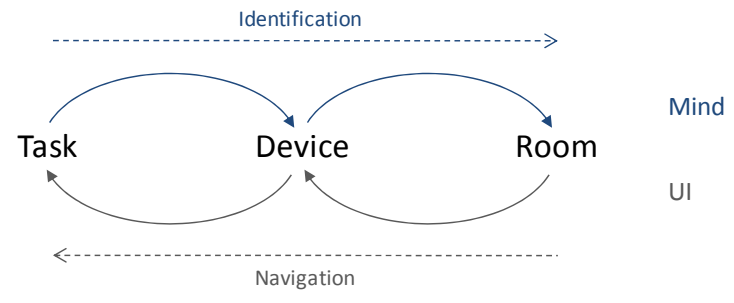

Figure 2. Performing an intended task

As our current target group of AAL are young elderly people living at home, proper candidates were people who were retired but still active in social activities, and who still live in their own homes, in particular, not in nursing homes. Therefore, we included a corresponding total of 13 women and men between 55 and 85 years of age, which we split into four groups with three, respectively four, participants. We provided each group with a set of 41 current or future services available in AAL environments (see Tasks in Figure 3$)^{2}$, which they should group into living room, kitchen, bathroom, bedroom, and hallway. The device level was omitted to simplify the setup, but almost every given task was unambiguously assignable to a specific device, since, for instance, the name of the device was part of the task itself ("Make a phone call"). The identified set of 41 services was printed on file cards, so that they could be easily handled (sorting, pushing, etc). Additional blue cards were provided in case the participants decided that some services did not fit into the given room groups. In that case, the participants should place these services into a new group and name that group appropriately. Finally, they were encouraged to think aloud, so that the moderator could comprehend and document the decisions made.

During this session, almost every group sorted in the same way, influenced, of course, by their personal habits (for example, whether they were used to making phone calls in the living room or in the hallway). As expected, each group used an additional group to place a set of tasks in. This group combined all services that were not unambiguously assignable to a single room, such as "Switch Light on/off" or "Check if all devices are switched off". Accordingly, this group was named "central", "everywhere", or similar by the participants. Finally, we named that group "House".

\footnotetext{
${ }^{1}$ In real use, this clustering will not be necessary, as a UCD can then adapt itself to the real apartment according to our metaphor,

${ }^{2}$ All materials used during both phases of our evaluation were originally in German. The prototype, examples, figures, comments by participants, or other results were especially translated into English for this publication.
} 
Based on the gathered data, we conducted a cluster analysis to identify certain communalities. For this purpose, the tool EZSort [12] was used to show the concrete relationship between pairs of services. The result of the closed cluster analysis is shown in Figure 3, symbolizing the relationships between pairs and their corresponding rooms. The shortest path between two services expresses the exact relationship, i.e., each participant put these services into the same group. So, the shorter this particular path is, the closer these two services are related to each other. However, as expected, we did not receive a $100 \%$ overlap for all tasks.

Using the results from the card sorting, a user interface prototype of a universal control device implementing the Apartment Metaphor was developed. However, before starting implementation, we first had to integrate the device level that was omitted before to simplify the setup. The service "Make a phone call", for instance, which was assigned directly to the hallway, is now, according to our Apartment Metaphor, assigned to a phone that is located in the hallway. This enables the suggested room-device-task navigation.

In particular, we had to take care of the services that could not be unambiguously assigned to rooms during the card sorting session and that were assigned to a new group by all participants as mentioned before. We decided to integrate an additional group "House", 3 which contains all devices that may affect the whole apartment. By selecting "Blinds", for instance, the user can control all blinds in her/his whole apartment. In addition, we also put most of these devices into every room. However, by selecting "Blinds" in a particular room, for instance, the user can only control the blinds in this room. Figure 4 shows an excerpt of the final task-device-room assignment.

For the implementation of our universal control device, we used the Adobe Flash [2] technology. In order to avoid negative effects on the results of our evaluation, we strongly adhered to guidelines regarding user interface design for elderly people, such as [5], [27], [36].

Moreover, we used photo-realistic instead of drawn icons to increase the recognizability of the devices. Later, the icons could also show a photo of the actual device in the apartment instead of a symbolic one. Also, to visualize the rooms, we used photo-realistic icons depicting typical stuff in a room (e.g., bed for bedroom). This seems to us more adequate for the elderly than an abstract visualization such as a floor plan. We decided to place these icons on a simple navigation bar at the bottom of the screen to "enter" a room. After entering, the upper area of the screen - named device selection area - then shows all available devices in this room (see Figure 5). If a concrete device is then selected, the device selection area will be replaced by a device-specific user interface, e.g., of a diary. The bar, however, always stays visible to enable the suggested two-click navigation. If the user wants to use another service, he/she only needs to (re)enter a room and select the corresponding device. Fortunately, this also allows us to leave out an explicit "Back" button, as we only use a navigational depth of two.

In order to provide a very intuitive interaction, the prototype was installed on a PaceBlade Slimbook P120 touchscreen TabletPC [24]. Thus, the participants were able to navigate by

\footnotetext{
${ }^{3}$ It appeared to be difficult to find an appropriate name and icon for that group. Although we were not really satisfied with the name "House", the participants liked it.
}

simply pressing the corresponding icons using their fingers, which has been proven as appropriate for elderly people [17].

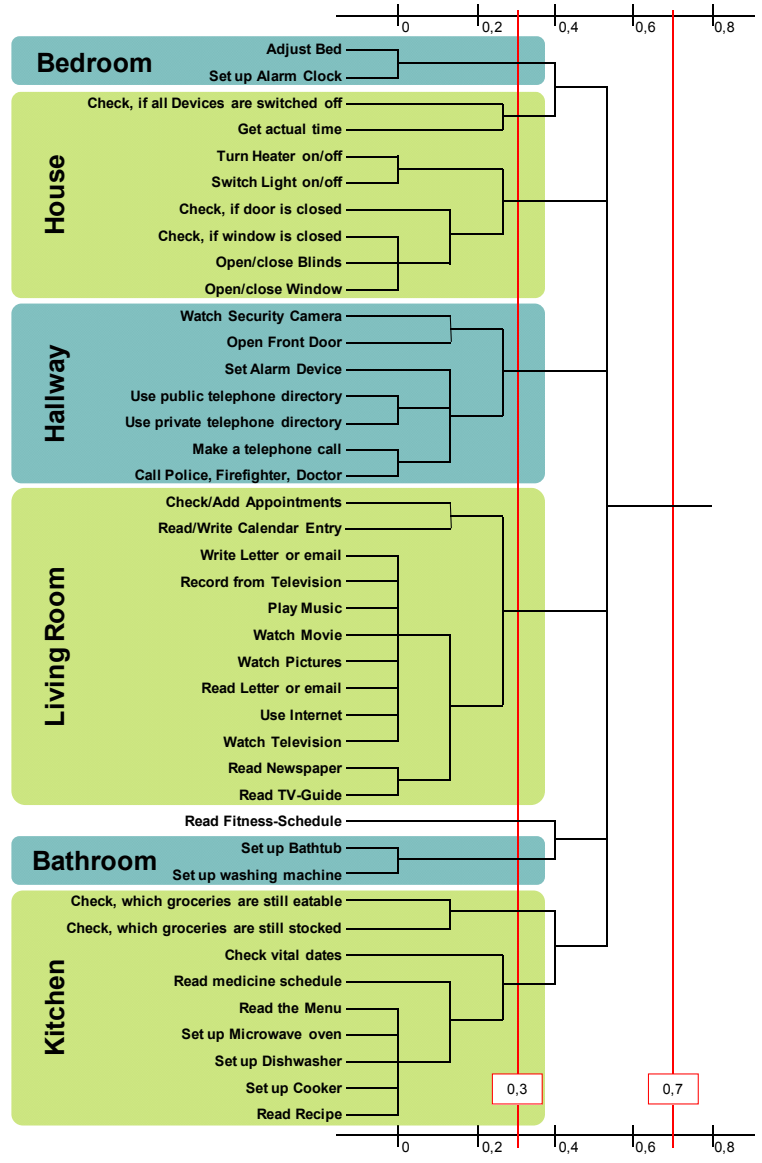

Figure 3. Cluster Analysis of the closed card sorting

Since we were only interested in whether and how the participants were able to find the corresponding service within the user interface (instead of performing the task itself), we left out any service functionality (and thus any device-specific user interface) at this prototype stage. After selecting a particular device, the user interface showed only textual information about the device's capabilities. Moreover, we left out the adaptable aspect considering the appearance or disappearance of the devices due to the already mentioned instant-messenger metaphor.

\section{EVALUATING THE APARTMENT METAPHOR}

Our main hypothesis is that the Apartment Metaphor is appropriate for universal control devices in the AAL domain. We evaluated this by testing the usability of the prototype's navigation structure in terms of effectiveness and efficiency according to metrics defined in [9].

\subsection{Hypotheses}

We broke down our main hypothesis into several subhypotheses addressing different aspects of a usable navigation structure.

\section{H1. Effectiveness - Completion rate:}

On average, at least $95 \%$ of the given task can be completed (by finding the corresponding device). 
Explanation: Our metaphor should enable elderly people to access all services available in an AAL environment. However, we estimate a default dropout rate of up to $5 \%$ which is always possible.

\section{H2. Effectiveness - Assistance:}

On average, at least $80 \%$ of the given task can be completed (by finding the corresponding device) without help.

Explanation: As mentioned before, the Apartment Metaphor should enable elderly people to access all available AAL services using one universal control device. However, due to the fact that the navigation structure in our prototype does not reflect the personal situation of each participant (which we will change in future work), we expect some participants to ask for help in situations where the navigation structure does not meet their expectations.

\section{H3. Efficiency - Click Count:}

The click rate to reach a corresponding service for a given task is distributed exponentially, with most tasks needing less than 2.5 clicks on average

Explanation: Following our metaphor, a user should need no more than 2 clicks on icons to choose a service: One to choose a room to which the device providing the service is associated, and another one to choose the device itself. Following this, we expect most tasks to be completed in about 2 clicks on average. Knowing about the problems described above, we also expected that some tasks would need more clicks.

\section{H4. Efficiency - Mean Time on Task:}

The time for reaching a corresponding service for a given task is distributed normally, with an average of 10 seconds and a standard deviation of 6 seconds.

Explanation: The mean time on task is expected to correlate with the click rate. The 10 seconds average is calculated on around 5 seconds per click. We consider this as a realistic value because the user interface and the test itself are completely new for the participants. However, as some tasks are more challenging than others, we expect a (standard) deviation of 3 seconds per click.

Besides these objective hypotheses, we also wanted to gather subjective reactions of the participants for better interpretation of the results. So, we were interested in the participants' opinion on how they feel (satisfaction) in order to get an impression on whether they had problems in using the navigation as intended (see Figure 2). However, we did not define any specific hypotheses for this.

\subsection{Sample}

As test candidates, we recruited group of young elderly participants that was similar to, yet different from the group used during the card sorting before. As the previous participants were already familiar with our idea of structuring tasks according to devices and rooms, it was important to select another set of individuals. The candidates, 10 men and 8 women between the age of 54 and 81 years with most being around 70 years old (see Figure 6), were selected randomly by an organization for elderly people in the city of Speyer, Germany. One third of them lived alone, while the other two thirds lived together with their partner (often one of the two was ill or partially disabled). $50 \%$ of them had a professional education; $50 \%$ even had a university degree. $77 \%$ had been retired for less than 10 years, and all were still active and taking part in social life. Their experience with personal computers reached from "no experience" to "daily work". The participants were volunteers and were not paid for their participation.

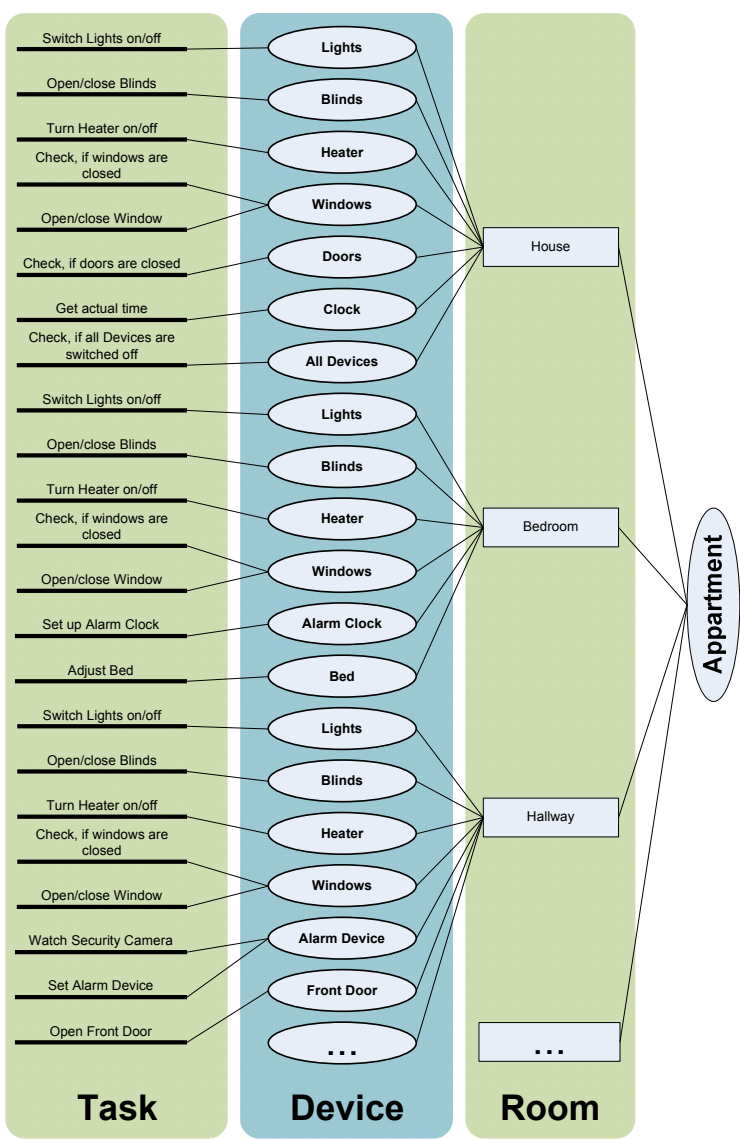

Figure 4. Excerpt from the task-device-room assignment

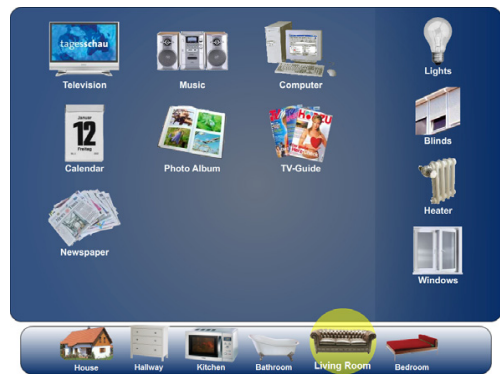

Figure 5. Screenshot from the prototype

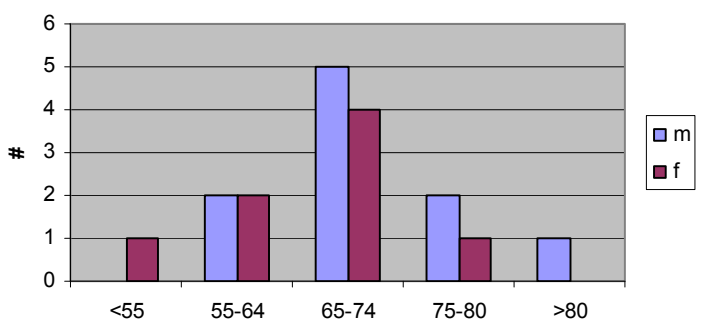

Figure 6. Age structure of the test candidates 


\subsection{Experimental Set-Up and Procedure}

As evaluation method we used a Usability Test [13]. For this purpose, we made use of a variety of technical devices executing and monitoring the whole situation. A video replay of the display output from the interaction device was recorded directly. Due to the haptic input, this could only be used to get the position where the participant actually pressed on the device. To get a complete replay of the navigation process including hesitation and hovering over icons without clicking, we used a camcorder (see Figure 7, left). To ensure privacy, we only recorded the hands instead of the whole participant. Both video streams were automatically synchronized and merged into a single stream (see Figure 7, right). Furthermore, a moderator and two observers, who noticed important situations, reactions and comments, were present. The moderator introduced the participants and explained them the purpose of the study, privacy issues, and the task to be done without giving concrete explanations that could corrupt the results. Moreover, he supported the participant during the test and encouraged them to go ahead we they started to hesitate. Again, the participants were explicitly asked to think aloud in order to get information about the way they feel and think.

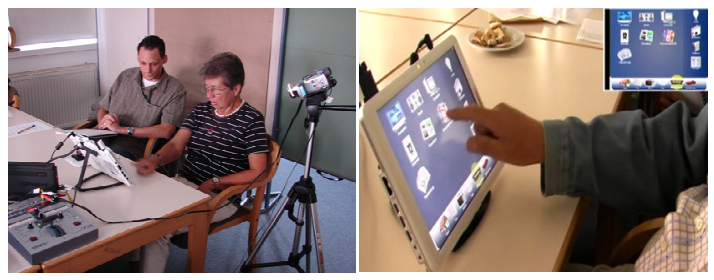

Figure 7. Participant during the evaluation

At the beginning of each run, the participant got a catalogue of 33 daily tasks, such as "Open a window", "Check your drug schedule", "Check if all devices are switched off", or "Listen to some music", which they should try to perform by using this prototype. These tasks had been identified by the consideration of the capabilities of remote controllable devices already existing on market or planed for the near future. The first tasks were quite self-explaining to achieve a positive feeling through quick success. The degree of difficulty constantly increased until the end of the catalogue was reached.

At the end of the experiment, a short interview was done with questions regarding satisfaction, feelings during the study, problems, opinions on the metaphor and prototype, and other questions of interest for our future research. Moreover, the participants were asked to fill out an AttrakDiff [14] questionnaire to measure the usability and attractiveness of the whole prototype.

\subsection{Analysis}

As results from the experiment, we got about 9 hours of video material from the observation and 18 filled-out interview and AttrakDiff questionnaires. To analyze the video material, we used the tool INTERACT [21], where it is possible to tag events, such as clicks, time intervals, such as the time for performing a task, and conditions, such as successful completion. The corresponding results were exported to a spreadsheet to perform statistical analysis.

Effectiveness: The first analysis concerned the effectiveness in terms of completion and assistance rate. On average, each participant completed $98 \%$ of the given tasks successfully (standard deviation 3\%). Only 3 persons could not achieve a
$95 \%$ completion rate, but still more than $90 \%$. Thus, we consider our hypothesis to be reliable. However, the participants could only complete an average of $86 \%$ (standard deviation 7\%) without help (see Figure 8). This analysis also shows that only 3 participants were unable to achieve the $80 \%$ completion without help.

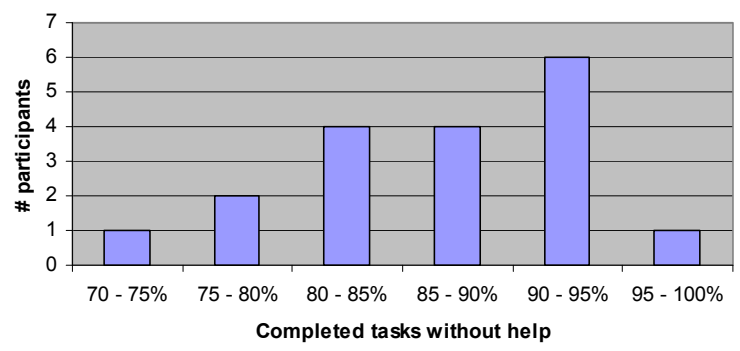

Figure 8. Task completion without help

Efficiency: With regard to efficiency, we analyzed the click rate and the mean time on task. Due to the above-mentioned reasons, the number of clicks and time needed to perform a task significantly differed between the tasks. The most problematic tasks we identified were five tasks in which the corresponding device could not be found in a unique room in all participants' homes (see Table 1). These tasks took more than 5.0 clicks on average. However, more than half of the tasks took less than 2.2 clicks on average and $48 \%$ of the tasks could even be completed with the minimum of 2 clicks. Figure 9 depicts the measured distribution of the click rate (without outliers). Testing this distribution shows that an exponential distribution can be assumed.

Table 1. List of the most problematic tasks

\begin{tabular}{|c|l|l|c|}
\hline$\#$ & \multicolumn{1}{|c|}{ Task } & \multicolumn{1}{c|}{$\begin{array}{c}\boldsymbol{\sigma} \\
\text { Clicks }\end{array}$} & $\begin{array}{c}\boldsymbol{\emptyset} \\
\text { Time }\end{array}$ \\
\hline 1 & $\begin{array}{l}\text { Look for your next doctor's } \\
\text { appointment. }\end{array}$ & 6.5 & $01: 15$ \\
\hline 2 & $\begin{array}{l}\text { Look at the wall clock to } \\
\text { see what time it is. }\end{array}$ & 6.5 & $00: 56$ \\
\hline 3 & Check your blood pressure. & 5.5 & $00: 52$ \\
\hline 4 & $\begin{array}{l}\text { Open the front door for the } \\
\text { postman. }\end{array}$ & 4.83 & $00: 43$ \\
\hline 5 & $\begin{array}{l}\text { Check if you have taken all } \\
\text { medications listed on your } \\
\text { medication plan. }\end{array}$ & 3.29 & $00: 32$ \\
\hline
\end{tabular}

This gap between "normal" and "problematic" task performance also becomes obvious when considering the mean time on task. The fastest tasks could, for instance, be completed in less than 4 seconds on average, while the slowest task took more than 75 seconds. Summarizing, a task was completed in an average of 16 seconds (with a standard deviation of 17 seconds), but when the five most problematic tasks are left out, average was 10 seconds with a standard deviation of 6 seconds - as expected. Testing this distribution shows that a normal distribution around the expected values can be assumed. 


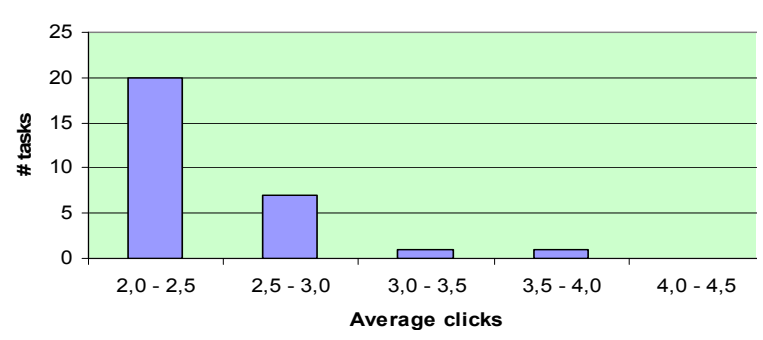

Figure 9. Clicks needed to perform the tasks

Satisfaction: The results from the post-interview were analyzed using a word processor for checking the free answers and comments, and a spreadsheet in which we noted the results of closed questions. The most interesting questions in this paper concerned two issues: First, the opinion of the participants with regard to the visualization of Apartment Metaphor elements; and second, the problems, if any, they had in using the navigation. Regarding the visualization of the metaphor elements, 94\% thought that most of the icons were comprehensible and that they did not have any problems clicking the right ones. These $94 \%$ were a very important value, as the success of a metaphor strongly depends on the visualization of its elements. If, for example, a user cannot understand the meaning of an icon, he would probably have problems finding the right services, even if he understood the underlying mental model.

With regard to the question of whether people had problems in using the navigation as intended (see Figure 2), $83 \%$ of the participants said that it was totally intuitive for them to identify an appropriate device for a given task. Furthermore, $78 \%$ of the participants did not experience any major problems when looking for a device in a room where they expected it. For most of them, only a few tasks, such as those listed in Table 1, were challenging, but in general, they knew where to look for a task-supporting device.

To analyze the results of the AttrakDiff questionnaire, we performed an automatic analysis using the free AttrakDiff tool [3]. The results showed that the participants were largely satisfied with the attractiveness / hedonic quality (HQ) and usability / pragmatic quality (PQ) of the prototype as a whole (see "P" box in Figure 10).

\section{INTERPRETATION}

The analysis of the objective measurements we got from the experiment largely confirmed our hypotheses.

Especially our hypotheses on efficiency could be exactly confirmed. This shows that user interfaces built upon our Apartment Metaphor can be used as efficiently as expected.

The fact that many services were reachable with exactly 2 clicks when using the prototypical device showed that the underlying assumption on how to use a user interface according to our metaphor (see Figure 2) was not far from reality. Especially the participants' reactions and statements observed during the evaluation (collected thanks to the participants' thinking aloud) lead to the assumption, that there was a mental mapping between tasks and corresponding devices and between devices and rooms they are typically located in. For instance, the task "Check what you need for the sauce" was commented by most participants by "Well, I go to the kitchen and take a look at the cookbook".

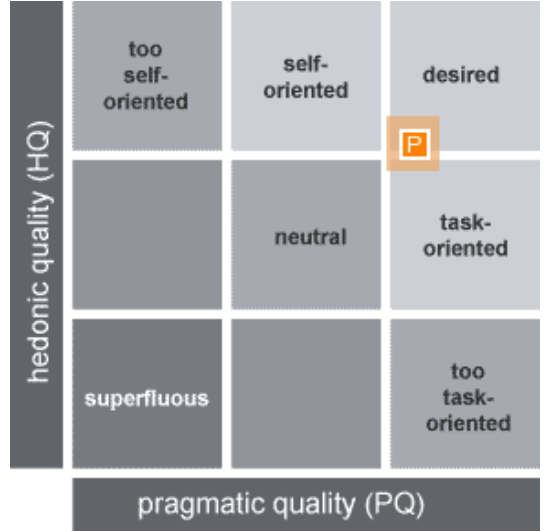

Figure 10. Result of AttrakDiff analysis

The problematic tasks that occurred can be traced back to the fact that the prototype was not personalized to the real participants' apartments. This led to some confused participants because a few devices were not available in a room where they expected them from their real-life experience, e.g., the phone that we grouped in the hallway based on the card sorting results, even though some people had it in the living room. These problematic tasks can thus be considered as the reason why we did not reach optimal values (such as $100 \%$ completion without help).

However, we are completely sure that these results do not influence the confirmation of the Apartment Metaphor in a negative way. Instead, we are convinced that many problems are an implicit confirmation of our metaphor. During the experiment we noticed that in the problematic cases, the participants always mentioned that they cannot find a device because it would be located in another room at their real home. This implies that they actually map the real apartment to the structure of the interface and that they navigate in the same way as they do it in real life: Identifying a device and the room it is located in and entering the room. Thus, the misguided navigation into the living room when looking for the phone, for instance, was a confirmation of our metaphor, if the participant's phone was located in her/his real living room (which we always asked in the problematic cases).

Even some participants recognized this problem and pointed out that in their opinion, there will be no one-fits-all solution and that the concrete user interfaces always has to be individually adapted to a specific apartment. Fortunately, this was something we already knew before. In a later productive environment, we therefore do not expect these problems, as the user interface will then be adapted to each user's habits.

\subsection{Threats to Validity}

The interpretation of our results requires an investigation of possible threats to validity. We distinguish between conclusion, internal, construct, and external validity.

\subsubsection{Conclusion Validity}

The quality of the gathered data can be considered as high, because the observations were recorded on video and monitored by two independent observers. Statistical instruments were used to calculate the significance of the hypotheses. The personal background of the participants regarding the experimental task was nearly the same, so we did not find any outlier that could corrupt the results. Also, the "problematic" tasks mentioned above did not influence our 
conclusion in a negative way, as we already expected these problems or as we ignored them during analysis.

\subsubsection{Internal Validity}

The independent variables remained unchanged between all participants. Disturbing factors during the performance of the experiment or during the analysis of the results were not observed. As the participants were volunteers, they were highly motivated and concentrated. Learning effects can be excluded, since every participant had only one session. Regarding the objective measures for effectiveness and efficiency, we therefore see no threats to the internal validity. However, regarding the subjective measures, such as the AttrakDiff questionnaire, we see a strong impact by some participants who were highly enthusiastic for life-assisting products due to personal need.

\subsubsection{Construct Validity}

The experiment was intended to show the appropriateness of the Apartment Metaphor. We defined the Apartment Metaphor as appropriate if and only if a navigation structure based on this metaphor is usable for the target group. To measure this usability, we took standardized metrics, formulated clear hypotheses, and selected 18 participants, who were asked to find 33 services within the navigation structure. Due to this, we consider the construction of our experiment as valid for its intended purpose.

\subsubsection{External Validity}

Even if we did not take cultural differences into consideration, the participants were a good and realistic representation of the target audience of AAL. Indeed, we did not assure that the distribution in sex, age, and education was completely representative of the elderly, but largely sufficient, as the participants were a real subset of the target audience. Especially with regard to the sustainability of the results, we selected young elderly around 70 years, as they will be the potential AAL users when this technology will actually come to market (in around 5 years). The environment of the experiment was a meeting room and not a real apartment. However, for the purpose of our investigation, this is not a threat. Thus, we expect to have high external validity.

\section{CONCLUSION}

The results of our investigation let us assume that the Apartment Metaphor is actually an appropriate aim to enable elderly people to access a large number of services available in an AAL environment in an intuitive way. The metaphor shows a way for structuring and visualizing services in a universal control device. Especially the 2-stage mapping from tasks to devices and the organization of these devices into virtual rooms that represent the real ones seem to be an adequate way for making AAL services accessible to the elderly. Therefore, our Apartment Metaphor proposes to structure universal control devices in AAL according to this mental model.

\section{FUTURE WORK}

The aim of the study we described in this paper was to show the impact of an Apartment Metaphor for the navigation structure and its visualization for universal control devices in (indoor) assisted living for young elderly people. However, we only showed that such a mental model is reasonable, without showing how services, devices and rooms were actually organized. The reason is that many decisions on how to assign devices to rooms are very personal decisions. Also, the problems observed during the experiment were only due to a structuring that did not meet the expectation and individual needs of each single participant. We are completely aware that for this reason there will not be a single one-fits-all solution. Instead, the structure must reflect the actual situation in a certain apartment. This can be done by manual personalization or automatic detection of available services and their location. In our future work, we will therefore extend our prototype with adaptive functionality and test it in our AAL lab [23].

Future investigations with regard to usability will consider scalability (i.e., how to make accessible a huge amount of devices that cannot be shown in a single view), usage-context adaptivity (i.e., whether it is reasonable to change the view when an event occurs in the apartment) and device migration (i.e., moving a device in a different room of the UCD when a physical device is moved into anther room). Our previous research regarding the structure and presentation of available services has been an important basis for this objective.

So far, we have only shown that our metaphor actually works. However, to better interpret this result, it is required that we compare Apartment Metaphor driven menus with other menu structures, for example those categorized by topics (e.g., medical, household, food etc). Consequently, we are currently preparing such a comparison.

\section{ACKNOWLEDGMENTS}

The work presented in this paper was carried out in the BelAmI (Bilateral German-Hungarian Research Collaboration on Ambient Intelligence Systems) project, funded by the German Federal Ministry of Education and Research (BMBF), the Fraunhofer-Gesellschaft, and the Ministry for Science, Education, Research and Culture (MWWFK) of RheinlandPfalz. Special thanks to Sabine Niebuhr, Claus Ritter, Kai Bizik, Christian Wiehr, and Ilhan Yilmaz for their support in the design and implementation of the evaluation study. We also thank all participants and the organization for elderly people in the city of Speyer, Germany, for coordinating them and providing rooms.

\section{REFERENCES}

[1] Aarts, E. and Encarnacao, J. L. Into Ambient Intelligence. True Visions - The Emergence of Ambient Intelligence, Springer, 2006.

[2] Adobe Flash. http://www.adobe.com/de/products/flash/ Last visited: 30.01 .2008 .

[3] AttrakDiff. http://www.attrakdiff.de. Last visited: 30.01 .2008 .

[4] Bomarius, F., Becker, M. and Kleinberger, T. Embedded intelligence for ambient assisted living. ERCIM News, 67, (2006), 19-20.

[5] Browne, H. Accessibility and Usability of Information Technology by the Elderly. http://www.otal.umd.edu/UUGuide/hbrowne/. Last visited: 30.01 .2008 .

[6] Card Sorting: A definitive guide. $\mathrm{http}: / /$ www.boxesandarrows.com/view/card_sorting_a definitive_guide. Last visited: 30.01.2008. 
[7] Card, S. K. and Henderson, A. A multiple, virtualworkspace interface to support user task switching. In Proc. CHI '87. ACM Press, 1987, 53-59.

[8] Card, S. K., Robertson, G. G. and Mackinlay, J. D. The information visualizer, an information workspace. In Proc CHI 1991. ACM Press, 1991, 181-186.

[9] Common Industry Format for Usability Tests, Version 2.0, May, 2001.

[10] Constantine, L. L. and Lockwood, L. A. Software for Use - A practical Guide to Models and Methods of Usage-Centered Design. Addison-Wesley, 1999.

[11] De Ruyter, B. Pelgrim, E. Ambient assisted-living research in carelab. Interactions 14, 4 (August 2007), 30-33.

[12] Dong, J., Martin, S., and Waldo, P. A user input and analysis tool for information architecture. Ext. Abstracts CHI 2001. ACM Press, 2001, 23-24.

[13] Dumas, J. and Redish, J. A practical guide to usability testing. Ablex Publishing, Norwood, 1993.

[14] Hassenzahl, M., Burmester, M. and Koller, F. AttrakDiff: Ein Fragebogen zur Messung wahrgenommener hedonischer und pragmatischer Qualität (A questionnaire for measuring the perceived hedonic and pragmatic quality). Mensch \& Computer (Human \& Computer). Teubner, 2003.

[15] Henderson, D. A. and Card, S. Rooms: the use of multiple virtual workspaces to reduce space contention in a window-based graphical user interface. $A C M$ Trans. Graph 5, 3, 1986.

[16] Hiroko, A. and Hiroyuki, M. Usability Research for the Elderly People. OKI Technical Review 199, 2004.

[17] Holzinger, A. Finger Instead of Mouse: Touch Screens as a Means of Enhancing Universal Access. User Interfaces for All, LNCS 2615. Springer, 2003, 387-397.

[18] Kuhn, W. and Blumenthal, B. Spatialization: Spatial Metaphors for User Interfaces. Conference Companion on Human Factors in Computing Systems CHI '96. ACM Press, 1996, 346-247.

[19] Lauesen, S. User Interface Design - A Software Engineering Perspective. Addison-Wesley, 2005.

[20] Lee, L. and Johnson, T., URCousin: Universal Remote Control User Interface. In Proceedings of the Human Interface Technologies Conference, 2006.

[21] Mangold International. http://www.mangold-international.com Last visited: 30.01 .2008 .
[22] Neale, D. C. and Carroll, J. M. The Role of Metaphors in User Interface Design. Handbook of HumanComputer Interaction. Elsevier, 1997, 441-462.

[23] Nehmer, J., Becker, M., Karshmer, A. and Lamm, R. Living assistance systems: an ambient intelligence approach. In Proc. ICSE '06. ACM Press, 2006, 43-50.

[24] PaceBlade Technology. http://www.paceblade.com Last visited: 30.01 .2008 .

[25] Remagnino, P. and Foresti, G.L. Ambient Intelligence: A New Multidisciplinary Paradigm. IEEE Transactions on Systems, Man and Cybernetics, Part A, 35, 1 (2005), 1-6.

[26] Sayago S. Some aspects of ICT accessibility, usability and design methods with the young elderly. Technical Report, Universitat Pompeu Fabra, Barcelona 2006.

[27] Shneiderman, B. Universal Usability. Communications of ACM, 43, 5 (2000), 84-91.

[28] Sjölinder, M., Höök, K. and Nilsson, L. Age differences in the use of an on-line grocery shop - implications for design. In Proc. CHI '00. ACM Press, 2000.

[29] Sjölinder, M., Höök, K. and Nilsson, L. Aging, cognition and task complexity in the use of an on-line grocery shop. Workshop on Web and Aging, HCI International 2003.

[30] Smith, D.C., Irby, C., Kimball, R. and Harslem, E. The star user interface: an overview. In Proc. AFIPS 55, (1986), 383-396.

[31] Stephanidis, C. User Interfaces for All: Concepts, Methods, and Tools. Laurence Erlbaum Associates, 2001.

[32] The Pebbles project. http://www.pebbles.hcii.cmu.edu/puc/ Last visited: 30.01 .2008 .

[33] The Universal Remote Console Consortium. http://myurc.org. Last visited: 30.01 .2008 .

[34] Vaananen K. and Schmidt, J. User Interface for Hypermedia: How to Find Good Metaphors? Conference Companion on Human Factors in Computing Systems CHI '94. ACM Press (1994), 263264.

[35] Weiser M. The computer for the 21st century. Scientific American, 1991.

[36] Zhao, H. Universal Usability Web Design Guidelines for the Elderly (Age 65 and Older), 2001. http://www.otal.umd.edu/UUPractice/elderly/ Last visited: 30.01 .2008 . 